\title{
Tunneling probe of fluctuating superconductivity in disordered thin films
}

\author{
David Dentelski, ${ }^{1,2}$ Aviad Frydman, ${ }^{1}$ Efrat Shimshoni, ${ }^{1}$ and Emanuele G. Dalla Torre ${ }^{1,2}$ \\ ${ }^{1}$ Department of Physics, Bar-Ilan University, 52900, Ramat Gan Israel \\ ${ }^{2}$ Center for Quantum Entanglement Science and Technology, Bar-Ilan University, 52900, Ramat Gan Israel
}

\begin{abstract}
Disordered thin films close to the superconducting-insulating phase transition (SIT) hold the key to understanding quantum phase transition in strongly correlated materials. The SIT is governed by superconducting quantum fluctuations, which can be revealed for example by tunneling measurements. These experiments detect a spectral gap, accompanied by suppressed coherence peaks that do not fit the BCS prediction. To explain these observations, we consider the effect of finite-range superconducting fluctuations on the density of states, focusing on the insulating side of the SIT. We perform a controlled diagrammatic resummation and derive analytic expressions for the tunneling differential conductance. We find that short-range superconducting fluctuations suppress the coherence peaks, even in the presence of long-range correlations. Our approach offers a quantitative description of existing measurements on disordered thin films and accounts for tunneling spectra with suppressed coherence peaks observed, for example, in the pseudo gap regime of high-temperature superconductors.
\end{abstract}

Introduction - Superconducting thin films have attracted recently a lot of attention due to the possibility of observing a direct superconductor-to-insulator transition (SIT) [1 3]. The SIT is considered as an excellent example of a quantum phase transition [4]: it occurs at temperature $T=0$ and is driven by a non-thermal tuning parameter $g$. Experimentally, the SIT can be driven by a wide variety of $g$, such as thickness [5-16, magnetic [11, 12, 17-26] or electric fields [27], chemical composition [28, 29], and disorder [25, 30]. Near the quantum critical point $g=g_{c}$, the system is governed by quantum fluctuations 31 35, and cannot be described in terms of classical Ginzburg-Landau theories [36-39].

In the vicinity of the SIT, experiments show an intriguing behavior of the superconducting energy gap $\Delta$. Traditionally, $\Delta$ is determined by fitting the tunneling conductivity to a phenomenological extension of the BCS theory, which takes into account an effective energy broadening $\Gamma$ and is known as the Dynes formula [40],

$$
\frac{d I}{d V}(V)=\operatorname{Re}\left[\frac{V-i \Gamma}{\sqrt{(V-i \Gamma)^{2}-\Delta^{2}}}\right] .
$$

This procedure is very useful in extracting values of $\Delta$ as a function of temperature for relatively clean superconductor. In these materials, $\Delta$ shrinks to zero as $T$ approaches the critical temperature $T_{c}$, in agreement to the predictions of the BCS theory [41]. In contrast, in disordered thin films, tunneling experiments have revealed that $\Delta$ smoothly evolves across the transition [42, 43, and through $T_{c}$ [44].

In addition to this non-conventional behavior of $\Delta$, superconducting thin films show a significant deviation of the experimentally measured DOS from Eq. (1), due to a considerable suppression of the coherence peaks at the gap edges [43, 44]. A similar disagreement was observed in high-temperature superconductors 45, 46. This discrepancy can be accounted for by assuming the two $\Gamma$ 's that appear in the numerator and in the denominator of
Eq. (1) to be different [47-49, but this approach lacks physical insight.

In this Letter we suggest an alternative approach which relates the experimental findings to a well defined theoretical model. Instead of considering an effective energy broadening, we include superconducting fluctuations by postulating a bosonic field $\Delta(\mathbf{r}, t)$ with a finite correlation length. By summing the contributions of short range and long range fluctuations, we obtain an excellent agreement with experimental curves on the insulating side of the SIT. Our results demonstrate that there are two important length scales: one is the effective size of a superconducting island, $\xi_{\mathrm{sc}}$, and the other is the typical size of quantum fluctuations $\xi_{\text {fluc }}$, which diverges at the SIT.

Superconducting fluctuations - We begin our analysis by introducing the Hamiltonian $H=H_{0}+H_{\Delta}$, where $H_{0}=\sum_{\mathbf{k}, \sigma} \varepsilon_{\mathbf{k}} c_{\mathbf{k}, \sigma}^{\dagger} c_{\mathbf{k}, \sigma}$ describes free electrons (quasiparticles) with a Fermi surface at $\varepsilon_{\mathbf{k}}=0$. Following Ref. [37, 38, we represent superconducting fluctuations by a randomly fluctuating bosonic field $\Delta(\mathbf{r}, t)$ coupled to the fermions by

$$
H_{\Delta}=\Delta(\mathbf{r}, t) c_{\uparrow}^{\dagger}(\mathbf{r}, t) c_{\downarrow}^{\dagger}(\mathbf{r}, t)+\text { H.c. . }
$$

In insulating samples, the superconducting order parameter averages to zero, $\langle\Delta(\mathbf{r}, t)\rangle=0$. We model finiterange superconducting fluctuations by a free field with two-point correlations

$$
C\left(\mathbf{r}-\mathbf{r}^{\prime}, t-t^{\prime}\right)=\left\langle\Delta(\mathbf{r}, t) \Delta^{*}\left(\mathbf{r}^{\prime}, t^{\prime}\right)\right\rangle .
$$

The function $C$ describes the decay of the superconducting correlations due to the interplay between disorder and interactions, and tends to zero at long distances and large times.

We now derive the relation between $C\left(\mathbf{r}-\mathbf{r}^{\prime}, t-t^{\prime}\right)$ and tunneling measurements. The tunneling differential conductivity is proportional to 50

$$
\frac{d I}{d V}(V) \propto \int_{-\infty}^{\infty} d \omega \rho(V+\omega) f^{\prime}(\omega) .
$$




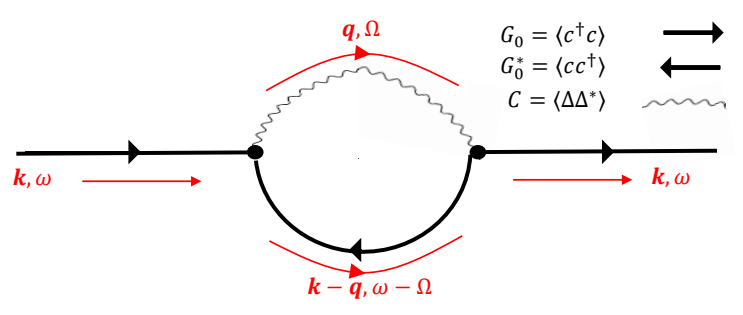

FIG. 1. One loop diagram - second order perturbation in $\Delta(\mathbf{r}, t)$. The black thick arrows represents charge (i.e. right arrow for a particle and left arrow for a hole), while the thin red ones represents momentum and energy.

Here $V$ is the voltage bias, $f^{\prime}(\omega)=d f / d \omega$ is the derivative of the Fermi-Dirac distribution function, and $\rho(\omega)$ is the density of states (DOS) of the sample. Eq. (4) assumes a constant DOS of the tip, and at $T=0$, simply reduces to $d I / d V \propto \rho(V)$. Within the Green's function formalism in Nambu space [51, $\rho(\omega)=$ $-(1 / \pi)\left\langle\operatorname{Im}\left\{\operatorname{Tr}\left[\sum_{\mathbf{k}} G^{r e t}(\mathbf{k}, \omega)\right]\right\}\right\rangle$, where $G^{r e t}(\mathbf{k}, \omega)$ is the retarded Green's function, Tr is the trace in Nambu space, Im is the imaginary part, and $\langle\ldots\rangle$ implies an average over the fluctuations of the superconducting field $\Delta(\mathbf{r}, t)$.

Our first step involves a Dyson resummation of the one-loop contributions shown in Fig. 1, whose two vertices represent the coupling term (2). By performing a trace over particle and hole contributions, we find

$$
\operatorname{Tr}\left[G^{r e t}(\mathbf{k}, \omega)\right]=\frac{\omega+i 0^{+}}{\left(\omega+i 0^{+}\right)^{2}-\varepsilon_{\mathbf{k}}^{2}-\mathcal{D}^{2}(\mathbf{k}, \omega)} .
$$

Here we defined the pairing-fluctuations function $\mathcal{D}$ as

$$
\mathcal{D}^{2}(\mathbf{k}, \omega)=\int d^{2} \mathbf{q} \int d \Omega \frac{\omega+i 0^{+}+\varepsilon_{\mathbf{k}}}{i \Omega+\omega+i 0^{+}+\varepsilon_{\mathbf{k}-\mathbf{q}}} C(\mathbf{q}, \Omega),
$$

with $C(\mathbf{q}, \Omega)=\int d^{2} \mathbf{r} d t C(\mathbf{r}, t) e^{i \mathbf{q} \cdot \mathbf{r}-i \Omega t}$.

Because the Green's function in Eq. (5) is strongly peaked at $\mathbf{k}=\mathbf{k}_{F}$, we can approximate the density of states as

$$
\rho(\omega)=\operatorname{Re}\left[\frac{\omega}{\sqrt{\omega^{2}-\mathcal{D}^{2}(\omega)}}\right],
$$

where we defined $\mathcal{D}(\omega) \equiv \mathcal{D}\left(\mathbf{k}_{\mathbf{F}}, \omega\right)$.

Eq. (7) is analogous to the Dynes equation (1), but involves the frequency dependent $\mathcal{D}(\omega)$ instead of the quasiparticle lifetime $\Gamma$. Note that if the correlation function $C(\mathbf{r}, t)$ does not decay in space and time (i.e. the BCS limit), its Fourier transform is $C(\mathbf{q}, \Omega) \sim \delta(\mathbf{q}) \delta(\Omega)$. In this case, Eq. (6) yields a frequency independent $\mathcal{D}^{2}(\omega)=\Delta_{0}^{2}$, and one recovers the well-known result.

Our approach has some similarities to Refs. [36 38, where superconducting fluctuations with a finite lifetime were considered as well. These authors were interested in the thermal regime $T \gg T_{c}$, where superconducting fluctuations are weak and lead to small deviations of the density of states. As a consequence, their approximation scheme does not recover the diverging density of states predicted by BCS. The Dyson resummation employed in the present work allows us to consider strong superconducting fluctuations and get closer to the SIT. See also Ref. [52] for a microscopic model describing the effect of superconducting fluctuations close to the SIT and their effects on the DOS.

In what follows, for simplicity, we will generically assume that the correlations are time independent, i.e.

$$
C(\mathbf{q}, \Omega)=C(\mathbf{q}) \delta(\Omega) .
$$

This assumption is justified if the collective-mode velocity $v$ is much smaller than the Fermi velocity $v_{F}$ : See the supplemental information for more details about the validity of this approximation. Assuming a quadratic dispersion relation $\varepsilon_{\mathbf{k}}=\left(k^{2}-k_{F}^{2}\right) v_{F} / 2 k_{F}$ and assuming that $C(\mathbf{q})$ decays to zero at $q \sim k_{F}$, we obtain

$$
\mathcal{D}^{2}(\omega)=\int d^{2} \mathbf{q} \frac{1}{\omega-v_{F} q_{x}} C(\mathbf{q}) .
$$

Eqs. (7) and (9) are the key theoretical results of our analysis, and we will now use them to model the density of states of disordered superconductors under various assumptions on the form of $C(\mathbf{q})$. In the following, the films are assumed to be thin enough such that both $\mathbf{k}$ and $\mathbf{q}$ can be treated as two dimensional.

Short range vs. long range - In order to understand the effects of superconducting fluctuations on the density of states, we consider correlation functions decaying over a typical inverse length scale $q_{0}$. This quantity can be associated with the average size of superconducting islands in the granular materials, and with an emergent electronic granularity of amorphous materials [31 35]. In the specific case of $C(\mathbf{q}) \sim \exp \left(-q^{2} / q_{0}^{2}\right)$, we can analytically solve the integral in Eq. (9) to find

$$
\mathcal{D}^{2}(\omega)=\Delta_{0}^{2} \frac{1}{v_{F} q_{0}} \exp \left(-\frac{\omega^{2}}{v_{F}^{2} q_{0}^{2}}\right) \omega\left[\operatorname{erfi}\left(\frac{\omega}{v_{F} q_{0}}\right)-i\right] .
$$

Here erfi is the imaginary error function, which is a real function. The real and imaginary parts of Eq. (10) are shown in the upper panel of Fig. 2(a-c) for different values of $v_{F} q_{0} / \Delta_{0}$, and the corresponding DOS in the lower panel. Note that the real part of $\mathcal{D}^{2}(\omega)$ closely resembles the local density of states $\rho(\omega)$, but these two quantities have a different physical meaning: the former actually needs to be substituted in Eq. (7) to deliver the latter. We observe that both $\operatorname{Re}\left[\mathcal{D}^{2}\right]$ and $\operatorname{Im}\left[\mathcal{D}^{2}\right]$ are peaked at a typical energy scale $v_{F} q_{0}$.

The effect of superconducting fluctuations on the DOS changes dramatically, depending on the ratio between 

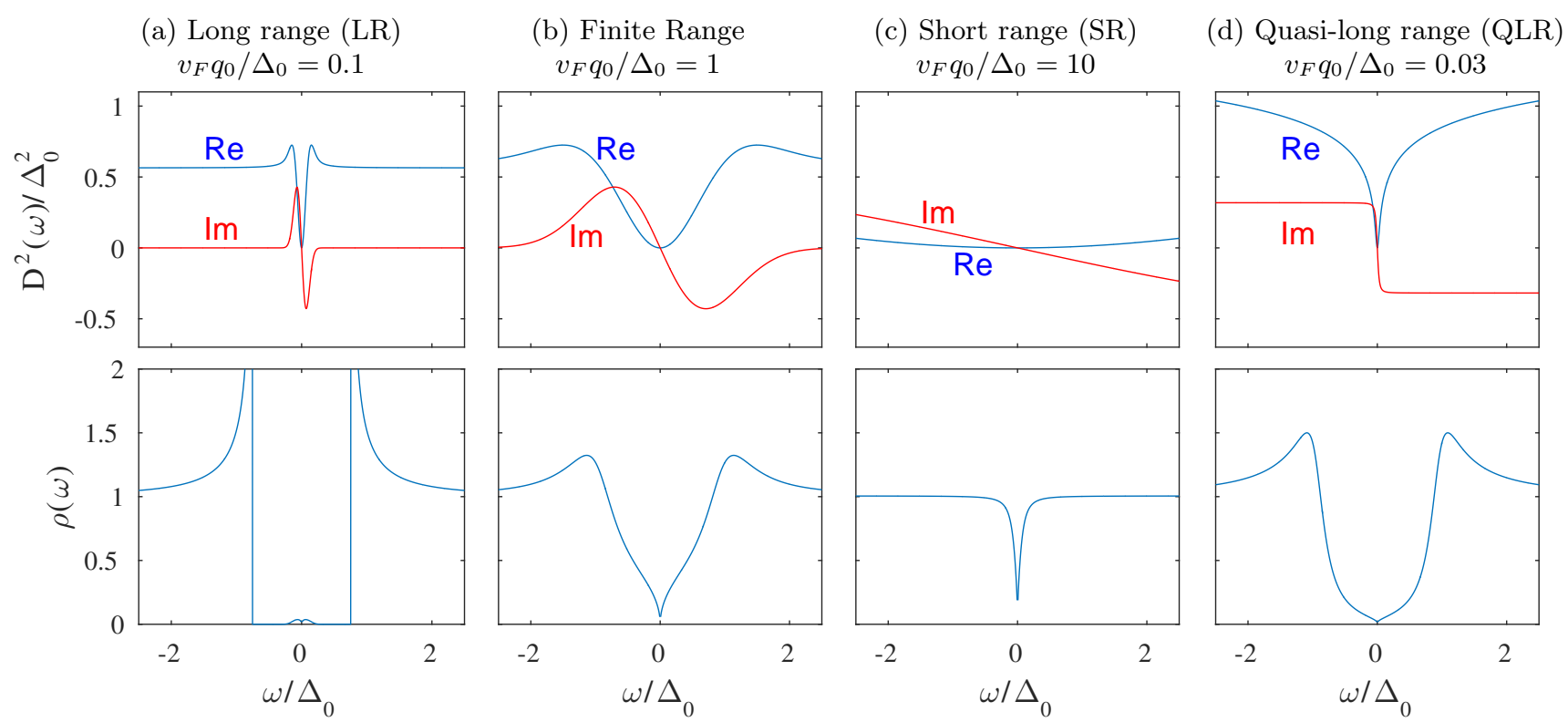

FIG. 2. Upper panel: (a-c) Real and imaginary part of Eq. 10p, for different values of $v_{F} q_{0} / \Delta_{0}$. (d) Real and imaginary part of Eq. 12]. Lower panel: the corresponding density of states, Eq. (7).

$v_{F} q_{0}$ and $\Delta_{0}$. Let us consider two extreme cases, which we denote by long range (LR) and short range (SR), respectively. The former case occurs for $v_{F} q_{0} \sim v_{F} \xi_{\text {fluc }}^{-1} \ll$ $\Delta_{0}$. In this case $\mathcal{D}^{2}(\omega)$ is approximately constant and we recover the BCS limit (see Fig. 2(a)). On the other hand, when $v_{F} q_{0} \sim v_{F} \xi_{\mathrm{sc}}^{-1} \gg \Delta_{0}$, the fluctuations are short ranged. In this regime

$$
\mathcal{D}^{2}(\omega) \approx-i \omega \gamma
$$

is purely imaginary, and the DOS shows a deep without coherence peaks (see Fig. 2(c)). The distinction between LR and SR fluctuations does not depend on the specific choice of $C(\mathbf{q})$, and can be related to the Anderson limit of superconductivity [53, 54. The crossover between these two regimes occurs when $\Delta_{0}$ is of the order of the typical energy level spacing of a superconducting island of size $1 / q_{0}$ (the superconducting correlation length), i.e. $\Delta_{0} \sim v_{F} q_{0}$.

In the vicinity of the SIT, superconducting fluctuations are described by a universal critical theory, which in its simplest form, is given by $C(\mathbf{q}) \sim 1 /\left(q^{2}+q_{0}^{2}\right)$ (See Ref. [55]), where $q_{0}=1 / \xi_{\text {fluc }}$ tends to zero at the transition. In this case, which we denote as quasi long range (QLR), we can again solve analytically Eq. (9) to obtain

$\mathcal{D}^{2}(\omega)=\frac{\Delta_{0}^{2}}{\pi^{2}} \frac{\omega}{\sqrt{v_{F}^{2} q_{0}^{2}+\omega^{2}}}\left[\ln \left(\frac{\sqrt{v_{F}^{2} q_{0}^{2}+\omega^{2}}+\omega}{\sqrt{v_{F}^{2} q_{0}^{2}+\omega^{2}}-\omega}\right)-i \pi\right]$

As shown in Fig.2(d), for $q_{0} \rightarrow 0$, the real part of Eq. (12) diverges logarithmically, while the imaginary part is proportional to $\operatorname{sign}(\omega)$. The resulting DOS resembles the LR situation and is very weakly dependent on the infrared cutoff $q_{0}$. As we will see below, QLR supercon- ducting fluctuations give a better description of the experiment than true LR correlations.

Comparison with experiments - In actual materials, one generically expects to find some combination of superconducting fluctuations with both long range and short range correlations. The former are universal and determine the emergent properties of the material, while the latter depend on the details of the materials and are often neglected. In contrast to this common practice, we find that short range correlation strongly affect the density of states (see Fig. 3): although the correlation functions denoted by LR and SR+LR have the same asymptotic behavior (subplot (a)), the corresponding DOS are very different (subplot (b)).

We now compare our theoretical calculations with the tunneling measurement of Ref. 43, performed on an $\mathrm{InO}$ film at $T=1 \mathrm{~K}$ at the insulating side of the SIT. Note that in order to isolate the superconducting contribution to the DOS, the experimental raw data was normalized by the tunneling spectra at high magnetic field. The results of our analysis are summarized in Table 1 and Fig. 4, where we show the best fitting parameters and the minimal normalized $\chi^{2}$-distribution between theory and experiment. We generically find that the sum of short range and long range superconducting fluctuations is required to obtain a good fit. Furthermore, a detailed analysis reveals that the long range part is best described by Eq. 12) (QLR, $\left.\chi^{2}=0.011\right)$, rather than Eq. 10p $\left(\mathrm{LR}, \chi^{2}=0.022\right)$, in agreement with the expected critical behavior of the SIT [55].

Discussion - In this Letter we studied the effects of superconducting fluctuations on the tunneling conductivity of disordered thin films, focusing on the insulating 
(a)

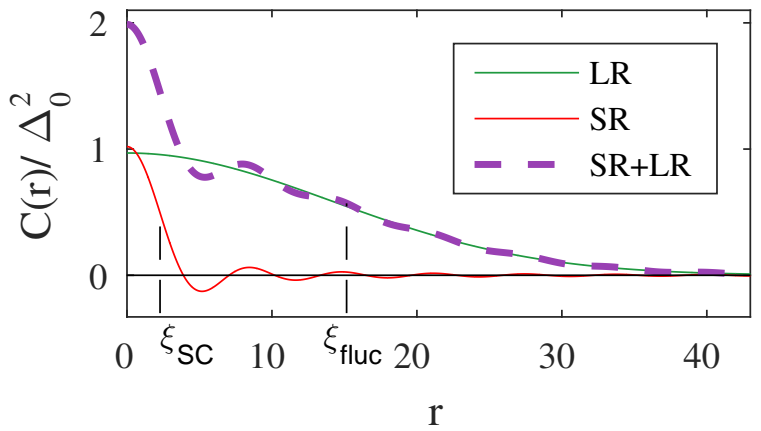

(b)

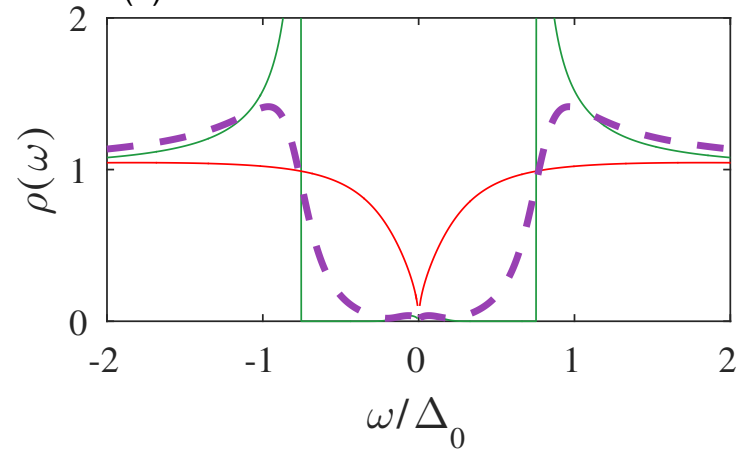

FIG. 3. (a) Normalized spatial correlations of the superconducting fluctuations, $C(r)$; (b) Corresponded density of states $\rho(\omega)$. Note that adding short range fluctuations (SR, $\left.v_{F} q_{0} / \Delta_{0}=3\right)$ suppresses the peaks, even in the presence of long range superconducting correlations (LR, $v_{F} q_{0} / \Delta_{0}=$ $0.1)$.

side of the SIT. The common approach, known as Dynes formula, relies on a phenomenological parameter $\Gamma$ that describes the inverse lifetime of the quasiparticles. In this study, we showed that the experiments are better fit by a theory of free electrons, coupled to superconducting fluctuations with finite-range correlations. By using a controlled diagrammatic approach, we derived a simple expression that connects the correlations of the superconducting fluctuations to the tunneling spectra, Eqs. (6) and (7). This result has potential applications that go beyond the present study, including quantum as well as classical superconducting phase transitions. Our analytical results show that, generically, short-range fluctuations lead to tunneling spectra with reduced or absent coherence peaks, even in the presence of long-range superconducting correlations.

By comparing our analytic expressions to experimental measurements, we find that in disordered thin films, the superconducting fluctuations are given by the sum of two components. The long range component is associated with universal fluctuations close to the SIT quantum critical point, characterized by a diverging length scale $\xi_{\text {fluc }}$. Accordingly, the experimental data is best fit by a critical theory with $q_{0} \sim 1 / \xi_{\text {fluc }} \ll k_{F}$ (see the last row of Table I. taking into account that $\left.v_{F} k_{F} \sim 1 \mathrm{eV}\right)$. In contrast, the short range component is determined by the micro-

\begin{tabular}{|c|c|c|c|}
\hline & DOS & best fit $[\mathrm{meV}]$ & $\chi^{2}$ \\
\hline Dynes & Eq. 11 & $\begin{array}{c}\Delta_{0}=0.73 \\
\Gamma=0.16\end{array}$ & 0.046 \\
\hline $\mathrm{SR}+\mathrm{LR}$ & $\begin{array}{c}\text { Eq. } 77 \text { with } \\
\left.\mathcal{D}^{2}(\omega)=\text { Eqs. } 11\right)+10\end{array}$ & $\begin{array}{c}\Delta_{0}=0.67 \\
\gamma=0.22 \\
v_{F} q_{0}=0.22\end{array}$ & 0.022 \\
\hline $\mathrm{SR}+\mathrm{QLR}$ & $\begin{array}{c}\text { Eq. } 77 \text { with } \\
\mathcal{D}^{2}(\omega)=\text { Eqs. } 11+12\end{array}$ & $\begin{array}{c}\Delta_{0}=0.69 \\
\gamma=0.11 \\
v_{F} q_{0}=0.022\end{array}$ & 0.011 \\
\hline
\end{tabular}

TABLE I. Comparison between different correlation function, i.e. Dynes, SR+LR and SR+QLR.

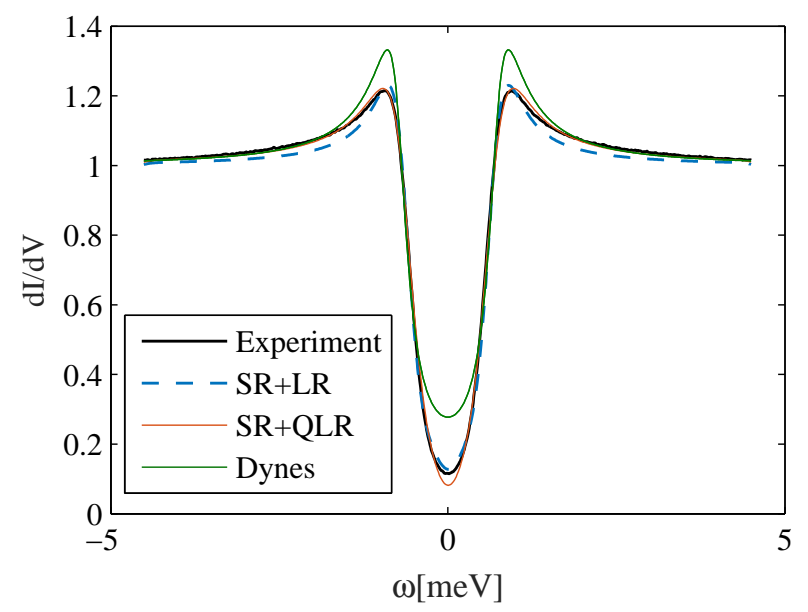

FIG. 4. Comparison between theoretical predictions and actual measurements performed on an insulating thin film close to the SIT. The best theoretical fits for each curve is presented at table I). The best fit to the experiment is given by $\mathrm{SR}+\mathrm{QLR}$.

scopic details of the material. Specifically, short range correlations are expected to play a predominant role in amorphous materials, where Cooper pairs are localized by disorder. In granular materials, on the other hand, the superconducting correlations decay over a much longer range, set by the typical scale of the grains. This distinction can explain why the Dynes formula (1) fits well experiments on granular $\mathrm{Pb}$ films 9, but does not fit amorphous InO films [4]. The distinction between short range and long range fluctuations can bridge the long standing controversy between the fermionic and bosonic approach to the SIT 54. On a broader prospective, our approach contributes to the understanding of puzzling spectrometric experiments in unconventional superconductors. Specifically, we find that although SR fluctuations contribute to the local superconducting gap, they generically lead to a tunneling spectrum with suppressed coherence peaks. When only SR fluctuations are present, the spectrum becomes v-shaped (see Fig. 2(c)), in analogy to the experimental observations in the pseudo-gap regime of underdoped cuprates (see for example Refs. [47, 48, 56, 57]). 
Acknowledgments - We thank Assa Auerbach, Igor Burmistrov, Tatiana Baturina, Oriya Eizenberg and Eugene Demler for useful discussions. This work is supported by the Israel Science Foundation, grant No. 1452/14 (E.G.D.T. and D.D.) and No. 231/14 (E.S.). A.F. acknowledges the support of the GIF foundation grant I-1250-303.10/2014. D.D. thanks the Klein family for a fellowship in memory of Prof. Michael Klein.

[1] Lin, Y.-H., Nelson, J. \& Goldman, A. Superconductivity of very thin films: The superconductor-insulator transition. Physica C: Superconductivity and its Applications 514, 130 (2015).

[2] Goldman, A. M. \& Markovic, N. Superconductorinsulator transitions in the two-dimensional limit. Physics Today 51, 39 (1998).

[3] Marković, N., Christiansen, C. \& Goldman, A. M. Thickness-magnetic field phase diagram at the superconductor-insulator transition in 2d. Phys. Rev. Lett. 81, 5217 (1998).

[4] Sachdev, S. Quantum Phase Transitions (Cambridge University Press, 2001).

[5] Strongin, M., Thompson, R., Kammerer, O. \& Crow, J. Destruction of superconductivity in disordered nearmonolayer films. Physical Review B 1, 1078 (1970).

[6] Dynes, R. C., White, A. E., Graybeal, J. M. \& Garno, J. p. Breakdown of eliashberg theory for two-dimensional superconductivity in the presence of disorder. Physical Review Letters 57, 2195 (1986).

[7] Haviland, D. B., Liu, Y. \& Goldman, A. M. Onset of superconductivity in the two-dimensional limit. Physical Review Letters 62, 2180 (1989).

[8] Valles Jr, J. M., Dynes, R. C. \& Garno, J. P. Electron tunneling determination of the order-parameter amplitude at the superconductor-insulator transition in $2 \mathrm{~d}$. Physical Review Letters 69, 3567 (1992).

[9] Merchant, L., Ostrick, J., Barber Jr, R. P. \& Dynes R. C Crossover from phase fluctuation to amplitudedominated superconductivity: A model system. Physical Review B 63, 134 (2001).

[10] Frydman, A., Naaman, O. \& Dynes, R. C. Universal transport in two-dimensional granular superconductors. Physical Review B 66, 052509 (2002).

[11] Hadacek, N., Sanquer, M. \& Villégier, J. C. Double reentrant superconductor-insulator transition in thin tin films. Physical Review B 69, 024505 (2004).

[12] Stewart, M. D., Yin, A., Xu, J. \& Valles, J. M. Superconducting pair correlations in an amorphous insulating nanohoneycomb film. Science 318, 1273 (2007).

[13] Sacépé, B., Chapelier, C., Baturina, T. I., Vinokur, V. M., Baklanov, M. R. \& Sanquer, M. Disorder-induced inhomogeneities of the superconducting state close to the superconductor-insulator transition. Physical Review Letters 101, 157006 (2008).

[14] Hollen, S. M., Nguyen, H. Q, Rudisaile, E., Stewart, M. D., Shainline,J., Xu, J. M \& Valles, J. M. Cooper-pair insulator phase in superconducting amorphous bi films induced by nanometer-scale thickness variations. Physical Review B 84, 064528 (2011).
[15] Baturina, T. I., Vinokur, V. M., Mironov, A. Yu., Chtchelkatchev, N. M., Nasimov, D. A. \& Latyshev, A. V. Nanopattern-stimulated superconductor-insulator transition in thin tin films. EPL (Europhysics Letters) 93, 47002 (2011).

[16] Poran, S., Nguyen-Duc, T.,Auerbach, A., Dupuis, N., Frydman, A. \& Bourgeois, O. Quantum criticality at the superconductor-insulator transition revealed by specific heat measurements. Nature Communications 8, 14464 (2017).

[17] Paalanen, M. A., Hebard, A. F. \& Ruel, R. R. Lowtemperature insulating phases of uniformly disordered two-dimensional superconductors. Physical Review Letters 69, 1604 (1992).

[18] Yazdani, A. \& Kapitulnik, A. Superconductinginsulating transition in two-dimensional a-moge thin films. Physical Review Letters 74, 3037 (1995).

[19] Gantmakher, V., Golubkov, M., Dolgopolov, V., Shashkin, A. \& Tsydynzhapov, G. Observation of the parallel-magnetic-field-induced superconductor-insulator transition in thin amorphous ino films. JETP Letters 71, 473 (2000).

[20] Sambandamurthy, G., Engel, L. W., Johansson, A. \& Shahar, D. Superconductivity-related insulating behavior. Physical Review Letters 92, 107005 (2004).

[21] Sambandamurthy, G., Engel, L. W., Johansson, A., Peled, E. \& Shahar, D. Experimental evidence for a collective insulating state in two-dimensional superconductors. Physical Review Letters 94, 017003 (2005).

[22] Steiner, M. A., Boebinger, G. \& Kapitulnik, A. Possible field-tuned superconductor-insulator transition in high-t c superconductors: Implications for pairing at high magnetic fields. Physical Review Letters 94, 107008 (2005).

[23] Baturina, T. I., Bentner, J., Strunk, C., Baklanov, M. R. \& Satta, A. From quantum corrections to magnetic-fieldtuned superconductor-insulator quantum phase transition in tin films. Physica B: Condensed Matter 359, 500 (2005).

[24] Baturina, T. I., Strunk, C., Baklanov, M. R. \& Satta, A. Quantum metallicity on the high-field side of the superconductor-insulator transition. Physical Review Letters 98, 127003 (2007).

[25] Crane, R. W., Armitage, N. P., Johansson, A., Sambandamurthy, G., Shahar, D., \& Gruner, G. Fluctuations, dissipation, and nonuniversal superfluid jumps in two-dimensional superconductors. Physical Review B 75, 094506 (2007).

[26] Vinokur, V. M., Baturina, T. I., Fistul, M. V., Mironov, A. Yu., Baklanov, M. R., \& Strunk, C. Superinsulator and quantum synchronization. Nature 452, 613 (2008).

[27] Parendo, K. A., Sarwa B. Tan, K. H., Bhattacharya, A., Eblen-Zayas, M., Staley, N. E. \& Goldman, A. M. Electrostatic tuning of the superconductor-insulator transition in two dimensions. Physical Review Letters 94, 197004 (2005).

[28] Mondal, M., Kamlapure, ., Chand,M., Saraswat, G., Kumar, S., Jesudasan, J., Benfatto, L., Tripathi, V. \& Raychaudhuri, P. Phase fluctuations in a strongly disordered s-wave nbn superconductor close to the metal-insulator transition. Physical Review Letters 106, 047001 (2011).

[29] Sherman, D. et al. The higgs mode in disordered superconductors close to a quantum phase transition. Nature Physics (2015).

[30] Poran, S., Shimshoni, E. \& Frydman, A. Disorder- 
induced superconducting ratchet effect in nanowires. Physical Review B 84, 014529 (2011).

[31] Shimshoni, E., Auerbach, A. \& Kapitulnik, A. Transport through quantum melts. Physical Review Letters 80, 3352 (1998).

[32] Bouadim, K., Loh, Y. L., Randeria, M. \& Trivedi, N. Single-and two-particle energy gaps across the disorder-driven superconductor-insulator transition. $\mathrm{Na}$ ture Physics 7, 884 (2011).

[33] Dubi, Y., Meir, Y. \& Avishai, Y. Nature of the superconductor-insulator transition in disordered superconductors. Nature 449, 876 (2007).

[34] Erez, A. \& Meir, Y. Thermal phase transition in twodimensional disordered superconductors. EPL 91, 47003 (2010).

[35] Erez, A. \& Meir, Y. Proposed measurement of spatial correlations at the berezinski-kosterlitz-thouless transition of superconducting thin films. Physical Review Letters 111, 187002 (2013).

[36] Cohen, R. W., Abeles, B. \& Fuselier, C. Effect of fluctuations in the superconducting order parameter on the tunneling density of states. Physical Review Letters 23, 377 (1969).

[37] Abrahams, E., Redi, M. \& Woo, J. W. Effect of fluctuations on electronic properties above the superconducting transition. Physical Review B 1, 208 (1970).

[38] Di Castro, C., Raimondi, R., Castellani, C. \& Varlamov, A. A. Superconductive fluctuations in the density of states and tunneling resistance in high- $T_{c}$ superconductors. Physical Review B 42, 10211 (1990).

[39] Varlamov, A., Balestrino, G., Milani, E. \& Livanov, D. The role of density of states fluctuations in the normal state properties of high t c superconductors. Advances in Physics 48, 655-783 (1999).

[40] Dynes, R. C., Narayanamurti, V. \& Garno, J. P. Direct measurement of quasiparticle-lifetime broadening in a strong-coupled superconductor. Phys. Rev. Lett. 41, 1509 (1978).

[41] Bardeen, J., Cooper, L. N. \& Schrieffer, J. R. Theory of superconductivity. Phys. Rev. 108, 1175 (1957).

[42] Barber Jr, R. P., Merchant, L. M., La Porta, A. \& Dynes, R. C. Tunneling into granular pb films in the superconducting and insulating regimes. Physical Review B 49, 3409 (1994).

[43] Sherman, D., Kopnov, G., Shahar, D. \& Frydman, A. Measurement of a superconducting energy gap in a homogeneously amorphous insulator. Physical Review Letters 108, 177006 (2012).

[44] Sacépé, B., Dubouchet, T., Chapelier, C., Sanquer, M., Ovadia, M., Shahar, D., Feigel'man, M. \& Ioffe, L. Localization of preformed cooper pairs in disordered superconductors. Nature Physics 7, 239 (2011).

[45] Zasadzinski, J., Tralshawala, N., Romano, P., Huang, Q., Chen, Jun. \& Gray, K. E. Tunneling density of states in cuprate and bismuthate superconductors. Journal of Physics and Chemistry of Solids 53, 1635 (1992).

[46] Dynes, R. The order parameter of high tc superconductors; experimental probes. Solid state communications 92, 53 (1994).

[47] Norman, M. R., Randeria, M., Ding, H. \& Campuzano, J. C. Phenomenology of the low-energy spectral function in high- $T_{c}$ superconductors. Physical Review B 57, R11093 (1998).

[48] Norman, M., Kaminski, A., Mesot, J. \& Campuzano,
J. Temperature evolution of the spectral peak in hightemperature superconductors. Physical Review B 63, 140508 (2001).

[49] Norman, M. R., Kanigel, A., Randeria, M., Chatterjee, U. \& Campuzano, J. C. Modeling the fermi arc in underdoped cuprates. Physical Review B 76, 174501 (2007).

[50] Mahan, G. Many-Particle Physics. Physics of Solids and Liquids (Springer, 2000).

[51] Altland, A. \& Simons, B. Condensed Matter Field Theory (Cambridge University Press, 2006).

[52] Burmistrov, I. S., Gornyi, I. V. \& Mirlin, A. D. Local density of states and its mesoscopic fluctuations near the transition to a superconducting state in disordered systems. Physical Review B 93, 205432 (2016).

[53] Anderson, P. W. Theory of dirty superconductors. Journal of Physics and Chemistry of Solids 11, 26 (1959).

[54] Seibold, G., Benfatto, L., Castellani, C. \& Lorenzana, J. Amplitude, density, and current correlations of strongly disordered superconductors. Physical Review B 92, 064512 (2015).

[55] Sondhi, S., Girvin, S., Carini, J. \& Shahar, D. Continuous quantum phase transitions. Reviews of Modern Physics 69, 315 (1997).

[56] Boyer, M. C., Wise, W. D., Chatterjee, K., Yi, M., Kondo, T., Takeuchi, T., Ikuta, H., \& Hudson, E. W., Imaging the two gaps of the high-temperature superconductor $\mathrm{Bi}_{2} \mathrm{Sr}_{2} \mathrm{CuO}_{6+\mathrm{x}}$. Nature Physics 3, 802 (2007).

[57] Fujita, K., Schmidt, A. R., Kim, E., Lawler, M. J., Lee, D. H., Davis, J. C., Hiroshi, E., \& Uchida, S., Spectroscopic imaging scanning tunneling microscopy studies of electronic structure in the superconducting and pseudogap phases of cuprate high- $T_{c}$ superconductors. Journal of the Physical Society of Japan 81, 011005 (2011). 


\section{SUPPLEMENTAL MATERIALS}

\section{TIME-DEPENDENT SUPERCONDUCTING FLUCTUATIONS}

The general form of the pairing fluctuation function, as presented in Eq. (6), contains an integral over the frequency $\Omega$. In the main text we considered correlation functions of the form $C(\mathbf{q}, \Omega)=C(\mathbf{q}) \delta(\Omega)$, for which the integral has a finite contribution only for $\Omega=0$. In this appendix we show numerically that our main findings are not changed significantly if the frequency dependence of $C$ is explicitly taken into account.

Following the assumptions mentioned in the main text, we approximate $\varepsilon_{\mathbf{k}-\mathbf{q}} \approx-\mathbf{k}_{F} \cdot \mathbf{q}=-v_{F} q \cos (\theta)$, where $\theta$ is the angle between $\mathbf{k}_{F}$ and $\mathbf{q}$, and obtain

$$
\begin{aligned}
\mathcal{D}^{2}(\omega)= & \int_{0}^{2 \pi} \int_{0}^{\infty} \int_{-\infty}^{\infty} d \Omega d q d \theta \times \\
& \frac{\omega q}{i \Omega+\omega-v_{F} q \cos (\theta)} C(q, \Omega) .
\end{aligned}
$$

The integral in Eq. S1) can be evaluated using the variable substitution $t=\tan \left(\frac{\theta}{2}\right)$, for which $\cos (\theta)=\frac{1-t^{2}}{1+t^{2}}$ and $d \theta=\frac{2}{1+t^{2}} d t$. Also, one needs to assume that $C(\mathbf{q}, \Omega)$ is $\theta$ independent, i.e. depends only on the absolute value of q. By introducing $A=q \omega C(q, \Omega), a=i \Omega+\omega$, and $b=v_{F} q$, we can rewrite the integral over $\theta$ as

$$
\begin{aligned}
& \int_{-\infty}^{\infty} d t \frac{2 A}{t^{2}(a+b)+a-b}= \\
& \int_{-\infty}^{\infty} d t \frac{2 A}{a+b} \frac{1}{(t+i \sqrt{m})(t-i \sqrt{m})}
\end{aligned}
$$

where $m=\frac{a-b}{a+b}$. By performing a standard Cauchy contour integral, we obtain

$$
\frac{2 \pi A}{\sqrt{(a+b)(a-b)}} \operatorname{sign}\left(\operatorname{Re} \sqrt{\frac{a-b}{a+b}}\right) .
$$

Recalling the original definition of $A, a$ and $b$, Eq. (6) can be written now as

$$
\begin{aligned}
& \mathcal{D}^{2}(\omega)= \int_{-\infty}^{\infty} \int_{0}^{\infty} d q d \Omega \times \\
& \frac{2 \pi q \omega C(q, \Omega)}{\sqrt{\left(i \Omega+\omega+v_{F} q\right)\left(i \Omega+\omega-v_{F} q\right)}} \times \\
& \operatorname{sign}\left(\operatorname{Re} \sqrt{\frac{i \Omega+\omega-v_{F} q}{i \Omega+\omega+v_{F} q}}\right) .
\end{aligned}
$$

To study the effects of a finite velocity of the collective excitations $v \neq 0$, we consider QLR fluctuations described by

$$
C_{Q L R}(q, \Omega)=\frac{A}{v^{2} q^{2}+v^{2} q_{Q L R}^{2}+\Omega^{2}}
$$

Here $v$ is the collective mode velocity and $A$ a normalization parameter. We next numerically compute $\mathcal{D}(\omega)$ from Eq. (S4), add the contributions from the SR correlations Eq. (11), and compare the resulting differential conductivity with the experiments. Using a derivative free optimization algorithm, we then minimize the $\chi^{2}$ difference between theory and experiment with respect to $\gamma, A, v, q_{0}$. The result of this minimization procedure gives an excellent agreement between theory and experiment $\left(\chi^{2}=0.007\right)$ : as shown in Fig. S1 the two curves are almost indistinguishable. This optimal fit is obtained for $k_{F} v=3.7 \mathrm{meV} \ll k_{F} v_{F} \sim 1 \mathrm{eV}$, confirming that $v \ll v_{F}$, as expected. This observation justifies the approximation $C(\mathbf{q}, \Omega) \approx C(\mathbf{q}) \delta(\Omega)$ used in the main text.

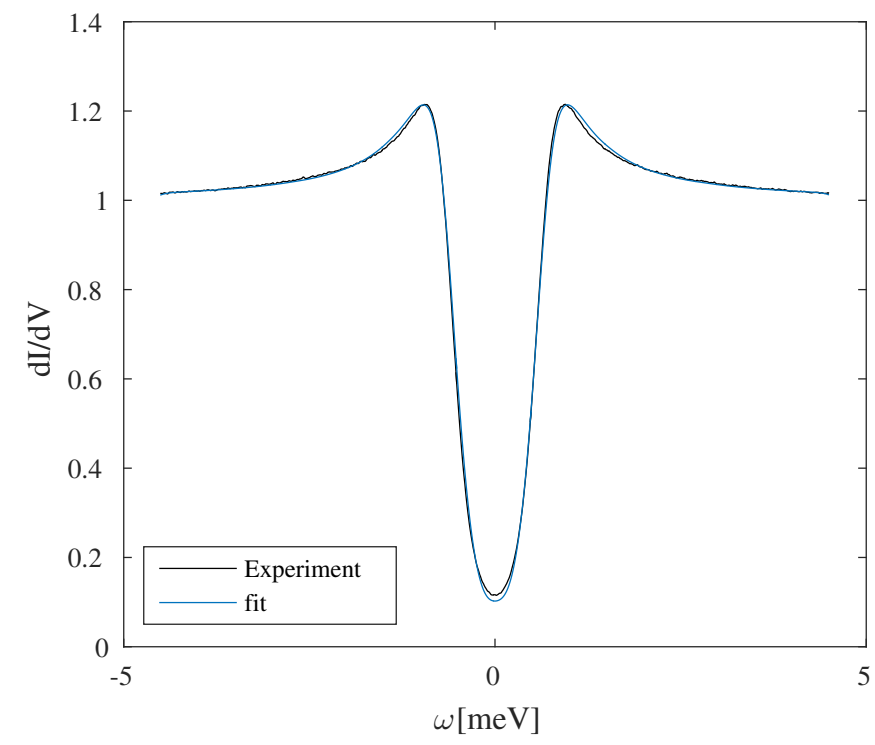

FIG. S1. Optimal fit of the DOS (Eq.7) using the sum of short range and time-dependent quasi long range superconducting fluctuations (Eqs. 111) and (S5) respectively). 


\section{DERIVATION OF THE DOS}

In the main text we introduced the Hamiltonian $H=H_{0}+H_{\Delta}$, where $H_{0}=\sum_{\mathbf{k}, \sigma} \varepsilon_{\mathbf{k}} c_{\mathbf{k}, \sigma}^{\dagger} c_{\mathbf{k}, \sigma}$ describes free electrons (quasiparticles) with a Fermi surface at $\varepsilon_{\mathbf{k}}=0$. We represented superconducting fluctuations by a randomly fluctuating bosonic field $\Delta(\mathbf{r}, t)$ coupled to the fermions by

$$
H_{\Delta}=\Delta(\mathbf{r}, t) c_{\uparrow}^{\dagger}(\mathbf{r}, t) c_{\downarrow}^{\dagger}(\mathbf{r}, t)+\text { H.c. . }
$$

In Nambu space, the bare Green's function corresponding to the free Hamiltonian is

$$
G_{0}^{-1}(\mathbf{k}, \omega)=\left(\begin{array}{cr}
i \omega-\varepsilon_{\mathbf{k}} & 0 \\
0 & i \omega+\varepsilon_{\mathbf{k}}
\end{array}\right)
$$

where $\omega \equiv \omega_{n}$, is the fermionic Matsubara frequency. The interaction matrix in Nambu space, which we denote by $V\left(\mathbf{k}-\mathbf{k}^{\prime}, \omega-\omega^{\prime}\right)$, is define according to $H_{\Delta}$ as:

$$
V\left(\mathbf{k}-\mathbf{k}^{\prime}, \omega-\omega^{\prime}\right)=\left(\begin{array}{cr}
0 & \Delta\left(\mathbf{k}-\mathbf{k}^{\prime}, \omega-\omega^{\prime}\right) \\
\Delta^{*}\left(\mathbf{k}-\mathbf{k}^{\prime}, \omega-\omega^{\prime}\right) & 0
\end{array}\right)
$$

Using the imaginary time path integrals formulation, and performing a standard resummation to obtain

$$
\mathcal{G}(\mathbf{k}, \omega)=\left[G_{0}^{-1}-\Sigma\right]^{-1}
$$

where $\Sigma=\sum_{\mathbf{k}^{\prime}, \mathbf{k}^{\prime \prime}, \omega^{\prime}, \omega^{\prime \prime}} V\left(\mathbf{k}-\mathbf{k}^{\prime}, \omega-\omega^{\prime}\right) G_{0}\left(\mathbf{k}^{\prime}, \omega^{\prime}\right) V\left(\mathbf{k}^{\prime}-\mathbf{k}^{\prime \prime}, \omega^{\prime}-\omega^{\prime \prime}\right)$ is the bubble of Fig. 1. We multiply both the numerator and denominator of Eq. (S9) by $G_{0}(\mathbf{k}, \omega)$ to obtain

$$
\mathcal{G}(\mathbf{k}, \omega)=\frac{G_{0}(\mathbf{k}, \omega)}{I-\sum_{\mathbf{k}^{\prime}, \mathbf{k}^{\prime \prime}, \omega^{\prime}, \omega^{\prime \prime}} V\left(\mathbf{k}-\mathbf{k}^{\prime}, \omega-\omega^{\prime}\right) G_{0}\left(\mathbf{k}^{\prime}, \omega^{\prime}\right) V\left(\mathbf{k}^{\prime \prime}-\mathbf{k}^{\prime}, \omega^{\prime \prime}-\omega^{\prime}\right) G_{0}(\mathbf{k}, \omega)}
$$

where $I$ is the 2X2 unit matrix. Using the defenition of the bare Green's function and interaction matrix, Eqs. S8 S9, the multiplication of $V\left(\mathbf{k}-\mathbf{k}^{\prime}, \omega-\omega^{\prime}\right) G_{0}\left(\mathbf{k}^{\prime}, \omega^{\prime}\right)$ gives

$$
\left(\begin{array}{cc}
0 & \frac{\Delta\left(\mathbf{k}-\mathbf{k}^{\prime}, \omega-\omega^{\prime}\right)}{\left(i \omega^{\prime}+\varepsilon_{\mathbf{k}^{\prime}}\right)} \\
\frac{\Delta^{*}\left(\mathbf{k}-\mathbf{k}^{\prime}, \omega-\omega^{\prime}\right)}{\left(i \omega^{\prime}-\varepsilon_{\mathbf{k}^{\prime}}\right)} & 0
\end{array}\right)
$$

The second multiplication is performed equivalently, where $\Delta\left(\mathbf{k}-\mathbf{k}^{\prime}, \omega-\omega^{\prime}\right) \rightarrow \Delta\left(\mathbf{k}^{\prime \prime}-\mathbf{k}^{\prime}, \omega^{\prime \prime}-\omega^{\prime}\right)$ and $\left(i \omega^{\prime} \pm \varepsilon_{\mathbf{k}}^{\prime}\right) \rightarrow$ $\left(i \omega \pm \varepsilon_{\mathbf{k}}\right)$.

Substituting Eq. S11 into Eq. S10 and taking the expectation value, we obtain:

$$
\mathcal{G}(\mathbf{k}, \omega)=G_{0}(\mathbf{k}, \omega)\left[I-\sum_{\mathbf{k}^{\prime}, \omega^{\prime}}\left(\begin{array}{cc}
\frac{C\left(\mathbf{k}-\mathbf{k}^{\prime}, \omega-\omega^{\prime}\right)}{\left(i \omega-\varepsilon_{\mathbf{k}}\right)\left(i \omega^{\prime}+\varepsilon_{\mathbf{k}^{\prime}}\right)} & 0 \\
0 & \frac{C\left(\mathbf{k}-\mathbf{k}^{\prime}, \omega-\omega^{\prime}\right)}{\left(i \omega+\varepsilon_{\mathbf{k}}\right)\left(i \omega^{\prime}-\varepsilon_{\mathbf{k}^{\prime}}\right)}
\end{array}\right)\right]^{-1},
$$

where $C\left(\mathbf{k}-\mathbf{k}^{\prime}, \omega-\omega^{\prime}\right) \delta\left(\mathbf{k}-\mathbf{k}^{\prime \prime}\right) \delta\left(\omega-\omega^{\prime \prime}\right)=\left\langle\Delta\left(\mathbf{k}-\mathbf{k}^{\prime}, \omega^{\prime}-\omega^{\prime \prime}\right) \Delta^{\star}\left(\mathbf{k}^{\prime \prime}-\mathbf{k}^{\prime}, \omega^{\prime}-\omega^{\prime \prime}\right)\right\rangle$ is the correlation function of the field $\Delta$. We can further simplify this expression as

$$
\mathcal{G}(\mathbf{k}, \omega)=G_{0}(\mathbf{k}, \omega)\left(\begin{array}{cc}
1-\sum_{\mathbf{q}, \Omega} \frac{C(\mathbf{q}, \Omega)}{\left[i \omega-\varepsilon_{\mathbf{k}}\right]\left[i(\omega-\Omega)+\varepsilon_{\mathbf{k}-\mathbf{q}]}\right.} & 0 \\
0 & 1-\sum_{\mathbf{q}, \Omega} \frac{C(\mathbf{q}, \Omega)}{\left[i \omega+\varepsilon_{\mathbf{k}}\right]\left[i(\omega-\Omega)-\varepsilon_{\mathbf{k}-\mathbf{q}]}\right.}
\end{array}\right)^{-1}
$$


where we define $\mathbf{k}-\mathbf{k}^{\prime}=\mathbf{q}$ and $\omega-\omega^{\prime}=\Omega$. We now invert the matrix and multiply it by $G_{0}(\mathbf{k}, \omega)$. Then, we perform an analytic continuation $i \omega \rightarrow \omega+i 0^{+}$and write the final result in the form of the retarded Green's function

$$
G^{r e t}(\mathbf{k}, \omega)=\left(\begin{array}{cc}
\frac{1}{\omega+i 0^{+}-\varepsilon_{\mathbf{k}}-\sum_{\mathbf{q}, \omega} \frac{C(\mathbf{q}, \Omega)}{\omega+i 0^{+}-i \Omega+\varepsilon_{\mathbf{k}-\mathbf{q}}}} & 0 \\
0 & \frac{1}{\omega+i 0^{+}+\varepsilon_{\mathbf{k}}-\sum_{\mathbf{q}, \omega} \frac{C(\mathbf{q}, \Omega)}{\omega+i 0^{+}-i \Omega-\varepsilon_{\mathbf{k}-\mathbf{q}}}}
\end{array}\right) .
$$

In order to write $G^{r e t}(\mathbf{k}, \omega)$ in a way which is similar to Dynes formula, we multiply the numerator and denominator of $G_{1,1}^{r e t}$ and $G_{2,2}^{r e t}$ by $\left(\omega+i 0^{+}\right) \pm \varepsilon_{\mathbf{k}}$ respectively. As a result

$$
G_{1,1}^{r e t}(\mathbf{k}, \omega)=\frac{\omega+i 0^{+}+\varepsilon_{\mathbf{k}}}{\left(\omega+i 0^{+}\right)^{2}-\varepsilon_{\mathbf{k}}^{2}-\sum_{\mathbf{q}, \Omega} \frac{\left(\omega+i 0^{+}+\varepsilon_{\mathbf{k}}\right) C(\mathbf{q}, \Omega)}{\omega+i 0^{+}-i \Omega+\varepsilon_{\mathbf{k}-\mathbf{q}}}},
$$

and

$$
G_{2,2}^{r e t}(\mathbf{k}, \omega)=\frac{\omega+i 0^{+}-\varepsilon_{\mathbf{k}}}{\left(\omega+i 0^{+}\right)^{2}-\varepsilon_{\mathbf{k}}^{2}-\sum_{\mathbf{q}, \Omega} \frac{\left(\omega-\varepsilon_{\mathbf{k}}\right) C(\mathbf{q}, \Omega)}{\left.\omega+i 0^{+}-i \Omega\right)-\varepsilon_{\mathbf{k}-\mathbf{q}}}} .
$$

The last term on each denominator was define in the main text as $\mathcal{D}^{2}$, and evaluated at $\mathbf{k}_{F}$ where $\varepsilon_{\mathbf{k}_{F}}=0$ (the justification can be found there). Thus, the two expressions are the same, except the sign of $\varepsilon_{\mathbf{k}-\mathbf{q}}$ in the denominator of $\mathcal{D}^{2}$. We approximate $\varepsilon_{\mathbf{k}_{F}-\mathbf{q}} \approx-\left(v_{F} / k_{F}\right)\left(\mathbf{k}_{F} \cdot \mathbf{q}\right)$ and take $\mathbf{q} \rightarrow-\mathbf{q}$ in Eq. (S16), so the denominator of both expression is equal. Consequently, after one perform an analytic continuation $i \omega \rightarrow \omega+i 0^{+}$the trace can be written as

$$
\operatorname{Tr}\left[\mathrm{G}^{\mathrm{ret}}(\mathbf{k}, \omega)\right]=\frac{\omega+\mathrm{i} 0^{+}}{\left(\omega+\mathrm{i} 0^{+}\right)^{2}-\varepsilon_{\mathbf{k}}^{2}-\mathcal{D}^{2}(\omega)}
$$

where

$$
\mathcal{D}^{2}(\omega)=\int d^{2} \mathbf{q} \int d \Omega \frac{\omega+i 0^{+}}{i \Omega+\omega+i 0^{+}+\varepsilon_{\mathbf{k}_{F}-\mathbf{q}}} C(\mathbf{q}, \Omega)
$$

